\title{
Pleural effusion during weaning from mechanical ventilation: a prospective observational multicenter study
}

\author{
Keyvan Razazi ${ }^{1,2,3^{*}} \mathbb{D}$, Florence Boissier ${ }^{4,5}$, Mathilde Neuville ${ }^{6}$, Sébastien Jochmans ${ }^{2,7}$, Martial Tchir ${ }^{8}$, Faten May ${ }^{1,2}$, \\ Nicolas de Prost ${ }^{1,2}$, Christian Brun-Buisson ${ }^{1,2}$, Guillaume Carteaux ${ }^{1,2}$ and Armand Mekontso Dessap ${ }^{1,2,3}$
}

\begin{abstract}
Background: Pleural effusion is common during invasive mechanical ventilation, but its role during weaning is unclear. We aimed at assessing the prevalence and risk factors for pleural effusion at initiation of weaning. We also assessed its impact on weaning outcomes and its evolution in patients with difficult weaning.

Methods: We performed a prospective multicenter study in five intensive care units in France. Two hundred and forty-nine patients were explored using ultrasonography. Presence of moderate-to-large pleural effusion (defined as a maximal interpleural distance $\geq 15 \mathrm{~mm}$ ) was assessed at weaning start and during difficult weaning.

Results: Seventy-three (29\%) patients failed weaning, including 46 (18\%) who failed the first spontaneous breathing trial (SBT) and 39 (16\%) who failed extubation. Moderate-to-large pleural effusion was detected in 81 (33\%) patients at weaning start. Moderate-to-large pleural effusion was associated with more failures of the first SBT [27 (33\%) vs. 19 $(11 \%), p<0.001]$, more weaning failures [37 (47\%) vs. $36(22 \%), p<0.001$ ], less ventilator-free days at day 28 [21 (5-24) vs. $23(16-26), p=0.01]$, and a higher mortality at day 28 [14 (17\%) vs. $14(8 \%), p=0.04]$. The association of pleural effusion with weaning failure persisted in multivariable analysis and sensitivity analyses. Short-term (48 h) fluid balance change was not associated with the evolution of interpleural distance in patients with difficult weaning.
\end{abstract}

Conclusions: In this multicenter observational study, pleural effusion was frequent during the weaning process and was associated with worse weaning outcomes.

Keywords: Mechanical ventilation, Pleural effusion, Weaning, Ultrasonography

\section{Introduction}

Several factors may contribute to the occurrence of pleural effusions in critically ill patients, including heart failure, pneumonia, hypoalbuminemia, and fluid overload [1]. Its incidence in mechanically ventilated patients varies depending on the screening method, from approximately $8 \%$ with physical examination to more than $60 \%$ with routine ultrasonography $[1,2]$. Pleural effusion was found in $83 \%$ of patients with acute respiratory distress

*Correspondence: keyvan.razazi@aphp.fr

1 AP-HP, DHU A-TVB, Service de Réanimation Médicale, Hôpitaux Universitaires Henri Mondor, 94010 Créteil, France

Full list of author information is available at the end of the article syndrome (ARDS) explored with computed tomography scans [3].

The presence of pleural effusion is associated with a longer duration of mechanical ventilation and intensive care unit (ICU) stay [2]. Although a causal relationship cannot be established, this prolongation may result from altered respiratory mechanics [4] and impeded diaphragmatic contraction [5]. Indeed, pleural effusion increases the total thoracic volume, leading inspiratory muscles to operate in a less advantageous portion of their length-tension curve. Thus, the capacity of the diaphragm to generate pressure decreases when pleural effusion increases [5, 6]. Drainage of large pleural effusions improves oxygenation and respiratory mechanics in mechanically ventilated patients $[4,7]$. 
Weaning accounts for approximately $40 \%$ of the total duration of mechanical ventilation [8], but data on pleural effusion during the weaning process are scarce [9]. The main objective of the present observational multicenter study was to assess the prevalence and risk factors of pleural effusion at initiation of weaning. The second objective was to explore the association of pleural effusion with weaning outcomes, and its evolution during difficult weaning.

\section{Materials and methods}

This prospective multicenter observational study recruited patients admitted in five ICUs in France. Inclusion criteria were endotracheal mechanical ventilation for at least $24 \mathrm{~h}$, and the fulfillment of weaning criteria [10] allowing a first spontaneous breathing trial (SBT). Noninclusion criteria were pregnancy or lactation, age less than 18 years, pleural effusion drainage before the first SBT, and a do-not-reintubate decision at time of inclusion.

\section{Weaning protocol and definitions}

Weaning initiation was defined as the day of first SBT. The first SBT used a T-piece trial in three centers and a low-level pressure support $\left(7-10 \mathrm{~cm} \mathrm{H}_{2} \mathrm{O}\right)$ with zero end-expiratory pressure in two centers, as per usual care. Failure of the SBT was based on predefined criteria (see the online supplement, Additional file 1). Extubation failure was defined as death or reintubation within the 7 days following extubation; this delay was used instead of $48-72 \mathrm{~h}$ because prophylactic noninvasive ventilation may postpone reintubation [11]. Indications for prophylactic noninvasive ventilation included patients older than 65 years and those with underlying cardiac or respiratory disease [12]. According to the International Consensus Conference [10], weaning success was defined as a first successful SBT followed by successful extubation. Failure of the weaning process was defined [10] as failure of the first SBT or extubation failure. Because some patients could not be classified with this definition, weaning was also categorized according to the WIND definition [13] as follows: short when the first SBT resulted in a successful termination of the weaning process or death within 1 day after the first SBT; difficult in case of successful weaning or death after more than 1 day but in less than 1 week after the first SBT; prolonged if weaning was still not terminated 7 days after the first SBT. Ventilator-free days at day 28 were computed as days without invasive mechanical ventilation during the 28 days following first SBT; patients who died before day 28 or were dependent on mechanical ventilation for more than 28 days after the first SBT had zero ventilator-free days [14]. Other definitions (e.g., Mac Cabe classification, ARDS, ventilator-associated pneumonia, failure of SBT) and data collection process are reported in the online supplement (Additional file 1).

\section{Lung ultrasonography}

Lung ultrasonography was performed on the day of first SBT and repeated on the 2 days following a SBT failure and on the day of extubation, if applicable. Maximal endexpiratory interpleural distance, sonographic patterns of effusion (homogeneously anechoic, complex nonseptated, complex septated, or homogeneously echogenic) [15], and of lung parenchyma (condensation or atelectasis) [16] were assessed on each side with the patient in the semirecumbent position. A moderate-to-large pleural effusion was defined as a maximal interpleural distance $\geq 15 \mathrm{~mm}$ (predicting an effusion volume of $300 \mathrm{~mL}$ or more) [17]; a large pleural effusion was defined by a maximal interpleural distance $\geq 25 \mathrm{~mm}[4,17]$. A pleural effusion was deemed drainable if the maximal interpleural distance was $\geq 15 \mathrm{~mm}$, and the effusion was visible over at least three intercostal spaces [18]. When possible, a transthoracic echocardiography was also performed to assess left ventricle ejection fraction (see the online supplement, Additional file 1). In patients with SBT failure, attempts at depletion (by diuretics or ultrafiltration) and fluid balance were collected during the 2 days following the SBT. There was no mandatory depletive strategy for the management of pleural effusion.

\section{Statistical analysis}

The primary endpoint was the prevalence of pleural effusion at weaning start. The sample size was calculated by hypothesizing a prevalence of pleural effusion of $40 \%$ [1-3], and considering a precision of $8 \%$. The study required a minimum of 170 patients (for an alpha risk of $5 \%$, i.e., a confidence interval of $95 \%$ ) and a maximum of 260 patients (for an alpha risk of $1 \%$, i.e., a confidence interval of $99 \%$ ). Continuous data were expressed as medians [25th-75th centiles] unless otherwise specified, and were compared using the Mann-Whitney test. Categorical variables, expressed as percentages, were compared using the Chi-square test or Fisher exact test. To evaluate independent factors associated with the presence of moderate-to-large pleural effusion at weaning start or with failure of the weaning process, significant or marginally significant $(p<0.10)$ bivariate risk factors (using the above mentioned tests) were examined using univariate and multivariable backward stepwise logistic regression analysis. Among related univariate factors, only the 
most statistically robust (yet clinically relevant) was entered into the regression model in order to minimize the effect of colinearity. The selection process was guided by consistency (less than $5 \%$ missing values) and maximal imbalances between groups (as estimated by absolute standardized differences, which are independent of the sample size and variable unit) [19]. Coefficients were computed by the method of maximum likelihood. The calibration of models was assessed by the Hosmer-Lemeshow goodness-of-fit statistic (good fit was defined as $p$ value $>0.05$ ), and discrimination was assessed by the area under the receiver operating characteristics curve (with a value of 1 indicating perfect discrimination, and a value of 0.5 indicating the effects of chance alone). Correlations were tested using the Spearman's method. Twotailed $p$ values $<0.05$ were considered significant. Data were analyzed using the IBM SPSS Statistics for Windows (Version 19.0, IBM Corp Armonk, NY, USA).

\section{Results}

\section{Study population}

The inclusion period lasted from 2 to 12 months depending on centers, between June 2015 and May 2016. Four hundred seventy-seven patients mechanically ventilated for more than $24 \mathrm{~h}$ were screened (Fig. 1). Sixty-seven patients died before weaning start, and 161 patients were excluded because of either a do-not-reintubate decision at time of inclusion $(n=72)$, unavailability of pleural ultrasound $(n=63)$, or drainage of pleural effusion before inclusion $(n=26)$. Thus, the present study comprises 249 patients assessed with lung ultrasonography at weaning initiation. Median duration of mechanical ventilation before weaning was 4 [2-7] days. The weaning trajectories are summarized in Fig. 1. Two hundred and three patients succeeded the first SBT, and 200 of them were extubated (the remainder three patients were not extubated despite the success of the first SBT because of borderline cough, and experienced a novel complication leading to death before any extubation attempt).

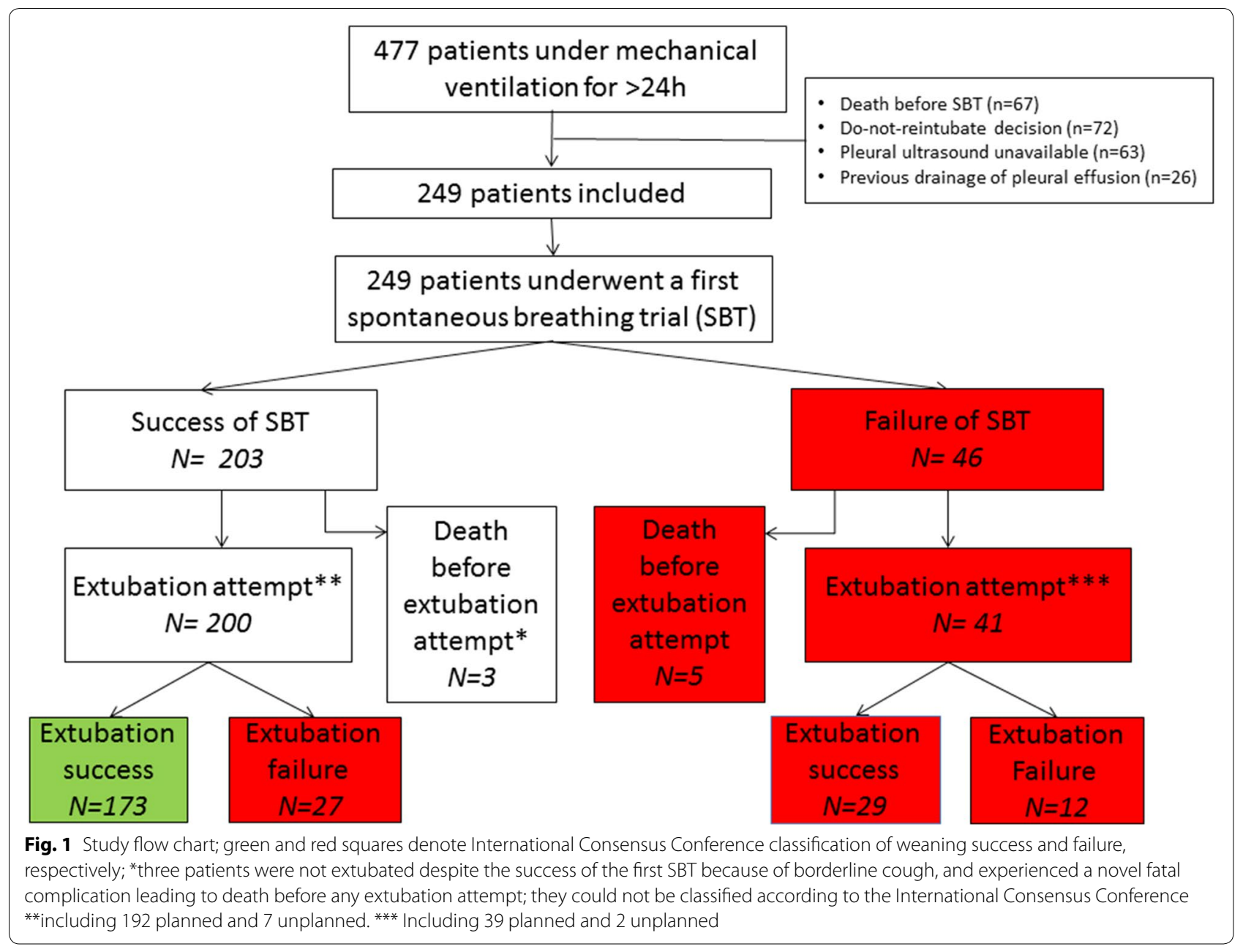


Forty-six patients (18\%) failed the first SBT; 41 of them succeeded a subsequent SBT and were extubated latter in the course of weaning, while the remainder five patients died before any extubation attempt. Reasons for SBT failure were respiratory rate $>35$ breaths/min with increased accessory muscle activity $(n=17), \mathrm{SpO}_{2}<90 \%$, while on $\mathrm{FiO}_{2} \geq 0.5(n=6)$, systolic blood pressure $<90 \mathrm{mmHg}$ or $>180 \mathrm{mmHg}(n=2)$, or a combination of those reasons $(n=22)$.

Overall, 241 patients were extubated during the weaning process, while eight patients died before any extubation attempt. Among the 241 patients extubated, 232 were planned and nine unplanned (including one accidental and eight self-extubations). After extubation, 95 (40\%) patients received noninvasive ventilation prophylactically, while twelve $(5 \%)$ received it for post-extubation acute respiratory failure. A total of $39(16 \%)$ patients failed extubation. The main reason for reintubation was acute respiratory failure $(n=23,73 \%)$.

\section{Prevalence and risk factors for pleural effusion}

A moderate-to-large pleural effusion was detected in 81 of 249 patients assessed at weaning initiation, for a prevalence of 33\%, 95\% confidence interval: $27-39 \%$. Most of pleural effusions were homogeneously anechoic $(n=74,93 \%)$ and associated with pulmonary condensation or atelectasis $(n=68,85 \%)$ (see the online supplement, Table e1, Additional file 1). Seventy-six (31\%) patients had a bilateral pleural effusion. The maximal interpleural distance was equally located either on the left $(n=41,51 \%)$ or right side $(n=40,49 \%)$. Patients with moderate-to-large pleural effusions at weaning initiation were older, had more baseline comorbidities and more organ failures before weaning as compared to their counterparts (see the online supplement, Table e4). In multivariable analysis, older age, McCabe class 2, cardiac disease, acute respiratory failure as cause of intubation, and need for dialysis before the first SBT were the five independent factors associated with a moderate-to-large pleural effusion at initiation of weaning (Table 1).

\section{Outcome of weaning}

According to the International Consensus Conference, the 249 patients were classified as follows: 173 (69\%) weaning successes (a first successful SBT followed by successful extubation); 76 (31\%) weaning failures (including 46 who failed the first SBT and 27 who succeeded the first SBT but failed extubation); three unclassifiable patients (despite the success of the first SBT, they were not extubated because of borderline

Table 1 Univariate and multivariable analysis of factors associated with moderate-to-large pleural effusion

\begin{tabular}{|c|c|c|c|c|}
\hline \multirow[t]{2}{*}{ Variables } & \multirow{2}{*}{$\begin{array}{l}\text { Missing } \\
\text { values, } n \\
(\%)\end{array}$} & \multirow{2}{*}{$\begin{array}{l}\text { Absolute } \\
\text { standardized } \\
\text { differences }\end{array}$} & \multicolumn{2}{|c|}{$\begin{array}{l}\text { Odd ratio ( } 95 \% \text { confidence interval), } p \text { value } \\
\text { by logistic regression }\end{array}$} \\
\hline & & & Univariate & Multivariable \\
\hline Age (per year) & 0 & 60.5 & $1.04(1.02-1.06), p<0.001$ & $1.03(1.01-1.05), p=0.017$ \\
\hline SAPS II (per point) & 0 & 26.9 & $1.02(1.0-1.03), p=0.048$ & $\mathrm{l} / \mathrm{NR}$ \\
\hline Mc Cabe class II (yes vs. no) & 0 & 51.8 & $4.7(2.1-10.3), p<0.001$ & $4.2(1.8-9.9), p=0.001$ \\
\hline Cancer or hematological malignancy (yes vs. no) & 0 & 47 & $3.5(1.7-7.2), p=0.001$ & $\mathrm{NI}$ \\
\hline Cardiac disease (yes vs. no) & 0 & 55.1 & $3.3(1.1-3.3), p<0.001$ & $2.2(1.1-4.4), p=0.02$ \\
\hline Left ventricle ejection fraction at cardiac ultrasound (\%), & $44(18 \%)$ & 52.9 & $0.96(0.93-0.98), p<0.001$ & $\mathrm{Nl}$ \\
\hline Supra-ventricular arrhythmias (yes vs. no) & 0 & 35.8 & $2.3(1.3-4.2), p=0.007$ & $\mathrm{NI}$ \\
\hline Acute respiratory failure as cause of intubation (yes vs. no) & 0 & 31.9 & $1.9(1.3-3.9), p=0.02$ & $1.8(0.98-3.2), p=0.059$ \\
\hline Dialysis (yes vs. no) & 0 & 31.5 & $2.5(1.2-5.4), p=0.02$ & $2.0(0.9-4.6), p=0.088$ \\
\hline Serum Creatinine (per $\mu \mathrm{mol} / \mathrm{L})$ & 0 & 25.8 & $1.0(0.99-1.00), p=0.20$ & $\mathrm{Nl}$ \\
\hline Septic shock (yes vs. no) & 0 & 25.4 & $1.7(0.98-2.9), p=0.06$ & $\mathrm{l} / \mathrm{NR}$ \\
\hline ARDS (yes vs. no) & 0 & 22.2 & $1.7(0.91-3.1), p=0.098$ & $\mathrm{l} / \mathrm{NR}$ \\
\hline Duration of MV before first SBT (per day) & 0 & 19 & $1.04(0.99-1.1), p=0.15$ & $\mathrm{NI}$ \\
\hline
\end{tabular}

SAPS // simplified acute physiology score, COPD chronic obstructive pulmonary disease, ARDS acute respiratory distress syndrome, SBT spontaneous breathing trial, NI not included, $I / N R$ included, but not retained by the final model

Among related univariate factors, only the most statistically robust (yet clinically relevant) was entered into the regression model in order to minimize the effect of colinearity. The selection process was guided by consistency (less than $5 \%$ missing values) and maximal imbalances between groups (as estimated by absolute standardized differences) as follows: Mc Cabe class II was selected among Mc Cabe class II, cancer and hematological malignancy; dialysis was selected among creatininemia and dialysis; cardiac disease was selected among supra-ventricular arrhythmias, left ventricle ejection fraction and cardiac disease; ARDS was selected among duration of mechanical ventilation before the first spontaneous breathing trial and ARDS before inclusion. The multivariable model showed a good calibration as assessed by the Hosmer and Lemeshow goodness-of-fit test $\left[X^{2}(8 \mathrm{~d} f)=6.42, p=0.60\right]$ and a fair discrimination as assessed by the receiver operating characteristics curve [area under the curve of $0.74(0.67-0.80), p<0.001$ ] 
cough, and experienced a novel complication leading to death before any extubation attempt) (Fig. 1). According to the WIND classification, 161 (65\%) patients had a short weaning, $60(24 \%)$ had a difficult weaning, and $28(11 \%)$ had a prolonged weaning. The presence of a moderate-to-large pleural effusion at weaning initiation was associated with more failures of the first SBT [27 (33\%) vs. $19(11 \%), p<0.001]$, more weaning failures [37 $(47 \%)$ vs. $36(22 \%), p<0.001]$, less ventilator-free days at day 28 (21 [5-24] vs. 23 [16-26], $p=0.01)$, and a higher mortality at day 28 [14 (17\%) vs. $14(8 \%), p=0.04]$ (Table 2, Fig. 2). All variables associated with weaning failure are shown in Table 3 and Table e5. In multivariable analysis, $\mathrm{PaO}_{2} / \mathrm{FiO}_{2}$ ratio, chronic obstructive pulmonary disease, a longer duration of mechanical ventilation prior to weaning, and the presence of moderate-to-large pleural effusion at weaning initiation were the four independent factors associated with weaning failure (Table 4). In sensitivity analyses, the association of pleural effusion with weaning failure also persisted after adjustment on SAPS II, in selected centers using the T-piece trial, or in those using a low-level pressure support, and when considering pleural effusions deemed drainable (as defined by a maximal interpleural distance $\geq 15 \mathrm{~mm}$ with the effusion visible over three intercostal spaces) or those considered large (as defined by a maximal interpleural distance $\geq 25 \mathrm{~mm}$ ) [4, 17] (Table e3). A moderate-to-large pleural effusion was detected in 60 (28\%) of 218 patients assessed on the day of first extubation attempt. The extubation failure rate was higher in patients with a moderate-to-large pleural effusion on the day of extubation as compared to their counterparts [14 (23\%) vs. 19 (12\%), $p=0.04$ ]; of note, this extubation failure rate was similar in patients with or without pleural effusion assessed earlier, at weaning initiation [24 (15\%) vs $15(20 \%), p=0.31$ ]. As compared to patients without effusion $(n=168)$, those with a unilateral $(n=21)$ or bilateral $(n=60)$ moderate-tolarge pleural effusion had similarly altered weaning outcomes, including SBT failure [19 (11.3\%) vs. 8 (38.1\%) vs. $19(31.7 \%), p<0.001]$ and weaning failure [37 $(22.0 \%)$ vs. $11(52.4 \%)$ vs. 28 (46.7\%), $p<0.001]$.

\section{Evolution of pleural effusion during difficult weaning}

Among the 46 patients who failed the first SBT, lung ultrasonography was repeated 24 and $48 \mathrm{~h}$ later in 41 and 31 patients, respectively. Patients in whom diuretics and/ or ultrafiltration were used had a lower fluid balance as compared with their counterparts $(-484[-1210-330]$ vs. 858 [205-1806] $\mathrm{mL}$ after $24 \mathrm{~h}, p<0.001)$, but this depletive strategy did not alter the interpleural distance (see the online supplement, Table e2, Additional file 1). Fluid balance was not significantly correlated with changes in interpleural distance $(\rho 0.13, p=0,17$, see Figure el of the online supplement, Additional file 2). Pleural effusion was drained in only four patients during weaning.

\section{Discussion}

We herein report the largest study assessing pleural effusion during weaning from mechanical ventilation. A moderate-to-large pleural effusion was detected by ultrasound examination in one-third of 249 patients at initiation of weaning and was associated with weaning failure by multivariable analysis. Depletive strategies did not alter pleural effusion volume on the short term in patients with difficult weaning.

\section{Prevalence and risk factors for pleural effusion}

In our study, one-third of patients had a moderate-tolarge pleural effusion at the initiation of the weaning process. This prevalence is higher than that of $13 \%$ reported by Dres et al. [9]. This discrepancy may be explained by differences in definitions used. Volume of pleural fluid was estimated in our report according to interpleural distance, which may be more sensitive than the classification of the British Thoracic Society [20] used in the latter study; indeed, patients with moderate-to-large pleural effusion in our study had a median interpleural distance inferior to the value found in the Dres' study (27 [20-41] vs. $45[30-60] \mathrm{mm})$. Other differences between these two studies include patient's comorbidities (with more patients included with cardiac diseases in our report) and/or timing of inclusion (with less patients excluded because of prior pleural drainage before SBT in our study).

Risk factors for pleural effusion found in our study are in accordance with previous reports $[1,2]$. Congestive heart failure is one of the leading factors associated with the occurrence of pleural effusion in ICU [1]. All patients intubated for acute respiratory failure had acute cardiac failure or pneumonia, two common risk factors for pleural effusion [1]. Our study suggests that diastolic dysfunction may be of importance in the association of cardiac failure with pleural effusion. Acute renal failure has also been previously reported as a risk factor for nonmalignant pleural effusions, an association possibly mediated by fluid overload [21]. The association of Mc Cabe class (i.e., a rapidly fatal underlying disease) with pleural effusion may be driven by other comorbidities like liver cirrhosis, cancer, and hypoalbuminemia $[22,23]$. 
Table 2 Characteristics and outcome of 249 mechanically ventilated patients with or without moderate-to-large pleural effusion at first spontaneous breathing trial

\begin{tabular}{|c|c|c|c|}
\hline \multirow{2}{*}{ Variables } & \multicolumn{2}{|c|}{ Moderate-to-large pleural effusion } & \multirow[t]{2}{*}{$p$ value } \\
\hline & Absent $(n=168)$ & Present $(n=81)$ & \\
\hline Male gender & $98(58 \%)$ & $52(64 \%)$ & 0.38 \\
\hline Age (years) & $61[50-72]$ & $69[60-80]$ & $<0.001$ \\
\hline SAPS II score at ICU admission & $49[37-62]$ & $52[41-67]$ & 0.07 \\
\hline \multicolumn{4}{|l|}{ Comorbidities } \\
\hline Neurological disease & $22(13 \%)$ & $6(7 \%)$ & 0.18 \\
\hline Cardiac disease & $93(55 \%)$ & $65(80 \%)$ & $<0.001$ \\
\hline Cirrhosis & $12(7 \%)$ & $9(11 \%)$ & 0.29 \\
\hline Chronic renal failure & $22(13 \%)$ & $16(20 \%)$ & 0.17 \\
\hline Cancer or hematological malignancy & $16(10 \%)$ & $22(27 \%)$ & $<0.001$ \\
\hline \multicolumn{4}{|l|}{ Main reason for intubation } \\
\hline Coma & $54(32 \%)$ & $9(11 \%)$ & $<0.001$ \\
\hline Acute respiratory failure & $51(30 \%)$ & $37(46 \%)$ & 0.02 \\
\hline Septic shock & $22(13 \%)$ & $12(15 \%)$ & 0.71 \\
\hline Others & $41(24 \%)$ & $23(28 \%)$ & 0.5 \\
\hline \multicolumn{4}{|l|}{ From ICU admission to first SBT } \\
\hline ARDS & $32(19 \%)$ & $23(28 \%)$ & 0.096 \\
\hline Duration of MV before the first SBT & $4[2-7]$ & $4[3-9]$ & 0.09 \\
\hline Dialysis & $15(9 \%)$ & $16(20 \%)$ & 0.015 \\
\hline \multicolumn{4}{|l|}{ Biological and ultrasound data at first SBT } \\
\hline Serum creatinine $(\mu \mathrm{mol} / \mathrm{L})$ & 74 [55-119] & 90 [60-164] & 0.07 \\
\hline Serum protide (mg/L) & $59[54-66]$ & 59 [51-63] & 0.19 \\
\hline Bilateral pleural effusion & $16(10 \%)$ & $60(74 \%)$ & $<0.001$ \\
\hline Maximal interpleural distance (mm) & $0[0-5]$ & $27[20-41]$ & $<0.001$ \\
\hline $\begin{array}{l}\text { Condensation or atelectasis of lung adjacent to the pleural } \\
\text { effusion at ultrasound }\end{array}$ & - & $68(84 \%)$ & \\
\hline Left ventricle ejection fraction (\%) & $60[50-60]$ & 50 [39-60] & $<0.001$ \\
\hline \multicolumn{4}{|l|}{ Outcome } \\
\hline Pleural effusion drainage during weaning & 0 & $4(5 \%)$ & 0.005 \\
\hline Prophylactic NIV post-extubation & $62(38 \%)$ & $33(43 \%)$ & 0.39 \\
\hline Failure of the first SBT & $19(11 \%)$ & $27(33 \%)$ & $<0.001$ \\
\hline Extubation failure & $24(15 \%)$ & $15(20 \%)$ & 0.31 \\
\hline Weaning failure ${ }^{\mathrm{a}}$ & $36(22 \%)$ & $37(47 \%)$ & $<0.001$ \\
\hline Weaning group ${ }^{b}$ & & & 0.03 \\
\hline Short weaning & $118(70 \%)$ & $43(53 \%)$ & \\
\hline Difficult weaning & $38(20 \%)$ & $26(32 \%)$ & \\
\hline Prolonged weaning & $16(10 \%)$ & $12(15 \%)$ & \\
\hline Tracheotomy & $4(2 \%)$ & $2(3 \%)$ & 0.97 \\
\hline VFD from first SBT to day 28 (days) & $23[16-26]$ & $21[5-24]$ & 0.01 \\
\hline Death in ICU & $14(8 \%)$ & $13(16 \%)$ & 0.07 \\
\hline Death at day 28 & $14(8 \%)$ & $14(17 \%)$ & 0.04 \\
\hline
\end{tabular}

Values are indicating number (\%) or median [1st-3rd quartile]

a According to the international conference consensus (three patients could not be classified)

${ }^{\mathrm{b}}$ According to the WIND study classification

SAPS II simplified acute physiology score, ARDS acute respiratory distress syndrome, SBT spontaneous breathing trial, NIV noninvasive ventilation, ICU intensive care unit, VFD ventilator-free days 


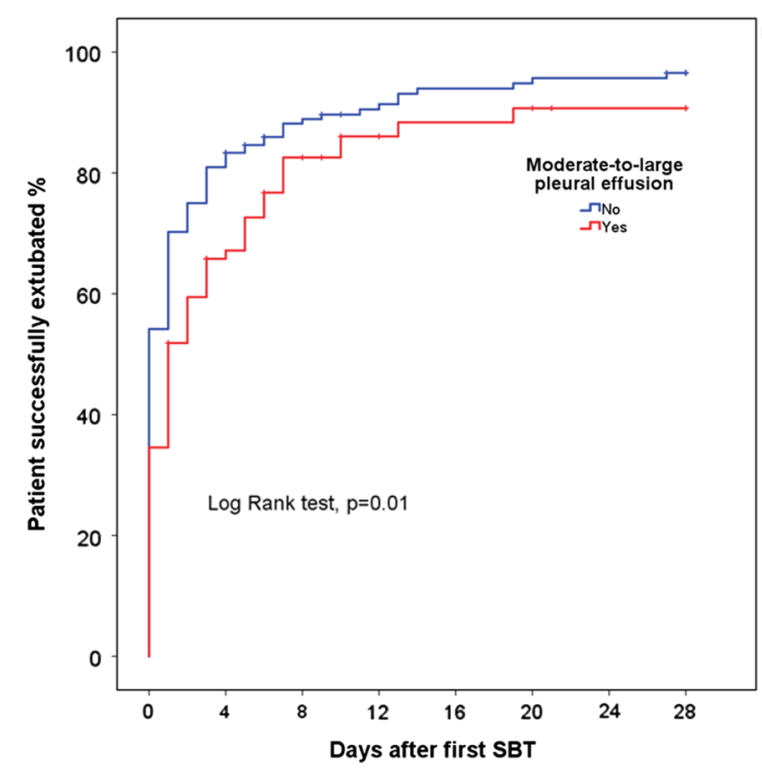

Fig. 2 Kaplan-Meier curves for the probability of successful extubation after the first spontaneous breathing trial in mechanically ventilated patients with (red lines) or without (blue lines) moderate-to-large pleural effusion

\section{Pleural effusion and weaning outcomes}

Our study is the first to show an association between moderate-to-large pleural effusion on the one hand and worse weaning outcomes and survival on the other hand. Dres et al. found similar weaning outcomes in patients with or without pleural effusion, but the limited number of patients with pleural effusions in their report $(n=18)$ weakened their conclusions [9]. Our findings are consistent with a previous report by Mattison et al., suggesting an association between pleural effusion and a longer duration of mechanical ventilation [2]. Although pleural effusion in patients with early ARDS do not seem to significantly influence lung physiology and gas exchange [3], its role in the latter stages of mechanical ventilation seems more relevant [4]. Physiological alterations associated with pleural effusion may worsen the respiratory load during the weaning process. Indeed, physiological studies showed that pleural effusion increases chest wall volume and decreases the length of inspiratory muscles and thus their efficiency and power $[5,6]$. Umbrello et al. recently showed that during weaning, drainage of a unilateral pleural effusion improves diaphragmatic contractile activity [24]. This improvement could decrease dyspnea and mitigate weaning failure. Pleural fluid accumulation may also result in relaxation atelectasis of the adjacent lung. In our study, most pleural effusions (85\%) were associated with condensation or atelectasis.

\section{Clinical implications}

There was no significant association between fluid balance and the evolution of pleural effusion during the $48 \mathrm{~h}$ following SBT failure. However, the negative fluid balance achieved was modest in the depletive group and this limitation precludes any definite conclusion. Pleural drainage with ultrasonography guidance has a low risk of complication under mechanical ventilator support [7] and may improve oxygenation, respiratory mechanics [4], and diaphragm performance [6, 24]. Removal of pleural fluid may therefore decrease the work of breathing and increase the ability of patients to succeed weaning. Further studies are needed to test whether a strategy of aggressive diuretic management or drainage of pleural effusions, in mechanically ventilated patients entering the weaning process, with others risk factors of weaning failure or a SBT failure, has the potential to decrease its duration [25].

\section{Strengths and limitations}

Strengths of our study include the large sample size, the prospective and multicentric design, and the use of ultrasound, which is currently considered the most sensitive method to detect pleural effusion at bedside. Our study has several limitations. First, only 249 of the 477 screened patients were included, a fact that may alter the external validity of our prevalence estimation. Second, the inter- or intra-observer agreement for pleural ultrasonography was not evaluated, but several reports previously demonstrated an excellent agreement for the measurement of left or right maximal interpleural distance [26]. Third, the physicians in charge of the patient were not fully blinded to the ultrasound examination results, and this may have theoretically influenced extubation decision and outcomes. However, criteria for SBT result were defined a priori and independent from ultrasound findings. Fourth, no estimation of respiratory drive (e.g., with airway occlusion pressure) nor respiratory muscle strength (e.g., with maximal inspiratory 
Table 3 Variables associated with weaning failure in $\mathbf{2 4 6}$ mechanically ventilated patients (three patients could not be classified according to the international conference consensus definition)

\begin{tabular}{|c|c|c|c|}
\hline Variables & Weaning success $(n=173)$ & Weaning failure $(n=73)$ & $p$ value \\
\hline Male gender & $98(57 \%)$ & $49(67 \%)$ & 0.13 \\
\hline Age (years) & $61[52-73]$ & 69 [60-79] & 0.006 \\
\hline Body mass index $\left(\mathrm{kg} / \mathrm{m}^{2}\right)$ & 26 [22-29] & 27 [22-32] & 0.07 \\
\hline SAPS II at ICU admission & $49[38-62]$ & 49 [39-65] & 0.73 \\
\hline \multicolumn{4}{|l|}{ Comorbidities } \\
\hline COPD & $23(13 \%)$ & $23(32 \%)$ & 0.001 \\
\hline Cardiac disease & $101(58 \%)$ & $55(75 \%)$ & 0.01 \\
\hline \multicolumn{4}{|l|}{ Main reason for intubation } \\
\hline Coma & $54(31 \%)$ & $9(12 \%)$ & 0.002 \\
\hline Acute respiratory failure & $48(27 \%)$ & $39(53 \%)$ & $<0.001$ \\
\hline Septic shock & $24(14 \%)$ & $8(11 \%)$ & 0.54 \\
\hline Others & $47(27 \%)$ & $17(23 \%)$ & 0.53 \\
\hline \multicolumn{4}{|l|}{ From ICU admission to first SBT } \\
\hline ARDS & $28(16 \%)$ & $27(37 \%)$ & $<0.001$ \\
\hline Neuromuscular blockade & $26(15 \%)$ & $28(38 \%)$ & $<0.001$ \\
\hline Septic shock & $61(35 \%)$ & $39(53 \%)$ & 0.01 \\
\hline VAP & $17(10 \%)$ & $16(22 \%)$ & 0.01 \\
\hline Supra-ventricular arrhythmias & $32(19 \%)$ & $22(30 \%)$ & 0.04 \\
\hline Duration of MV before first SBT & $3[2-6]$ & $6[3-12]$ & $<0.001$ \\
\hline Dialysis & $22(13 \%)$ & $8(11 \%)$ & 0.70 \\
\hline Fluid balance between ICU admission and first SBT (L) & $2.8[0.9-6.4]$ & $5.7[0.7-11.4]$ & 0.01 \\
\hline \multicolumn{4}{|l|}{ Biological and ultrasound data at first SBT } \\
\hline $\mathrm{PaO}_{2} / \mathrm{FiO}_{2}$ ratio $(\mathrm{mmHg})$ & 307 [242-385] & 247 [200-299] & $<0.001$ \\
\hline Moderate-to-large pleural effusion & $42(24 \%)$ & $37(51 \%)$ & $<0.001$ \\
\hline Drainable pleural effusion & $36(21 \%)$ & $29(40 \%)$ & 0.002 \\
\hline Large pleural effusion & $21(12 \%)$ & $25(34 \%)$ & $<0.001$ \\
\hline Left ventricle ejection fraction $(\%, n=205)$ & $60[50-60]$ & $55[40-60]$ & 0.06 \\
\hline \multicolumn{4}{|l|}{ Outcome } \\
\hline Pleural effusion drainage during weaning & 0 & $4(6 \%)$ & 0.01 \\
\hline Prophylactic NIV post-extubation & $65(38 \%)$ & $30(44 \%)$ & 0.35 \\
\hline Tracheotomy & $1(1 \%)$ & $5(7 \%)$ & 0.01 \\
\hline VFD from first SBT to day 28 (days) & $23[20-26]$ & $11[0-21]$ & $<0.001$ \\
\hline Death in ICU & $5(3 \%)$ & $19(26 \%)$ & $<0.001$ \\
\hline Death at day 28 & $8(5 \%)$ & $17(23 \%)$ & $<0.001$ \\
\hline
\end{tabular}

Values are indicating number (\%), or median [1st-3rd quartile]

SAPS II simplified acute physiology score, COPD chronic obstructive pulmonary disease, ARDS acute respiratory distress syndrome, VAP ventilator-associated pneumonia, SBT spontaneous breathing trial, NIV noninvasive ventilation, ICU intensive care unit, VFD ventilator-free days

pressures) was performed. Last, a significant decrease in pleural effusion during difficult weaning may have required more time, and/or more intense depletive fluid management [27].

\section{Conclusion}

Moderate-to-large pleural effusion was found in onethird of patients at initiation of weaning and associated with worse outcomes. Depletive strategies did not rapidly alter its evolution. Further studies should test the clinical 
Table 4 Univariate and multivariable logistic regression of factors associated with weaning failure $(n=246)$

\begin{tabular}{|c|c|c|c|c|}
\hline \multirow[t]{2}{*}{ Variables } & \multirow{2}{*}{$\begin{array}{l}\text { Missing } \\
\text { values, } \mathrm{n} \\
(\%)\end{array}$} & \multirow{2}{*}{$\begin{array}{l}\text { Absolute } \\
\text { standardized } \\
\text { differences }\end{array}$} & \multicolumn{2}{|c|}{$\begin{array}{l}\text { Odd ratio ( } 95 \% \text { confidence interval), } p \text { value } \\
\text { by logistic regression }\end{array}$} \\
\hline & & & Univariate & Multivariable \\
\hline Age (per year) & 0 & 47 & $1.03(1.01-1.05), p=0.01$ & $1.02(0.997-1.05), p=0.08$ \\
\hline Body mass index (per kg/m²) & $6(2 \%)$ & 32 & $1.06(1.01-1.11), p=0.02$ & I/NR \\
\hline COPD (yes vs. no) & 0 & 48 & $3.0(1.6-5.8), p=0.001$ & $2.2(1.02-4.7), p=0.045$ \\
\hline Cardiac disease (yes vs. no) & 0 & 37 & $2.2(1.2-4.0), p=0.01$ & I/NR \\
\hline Left ventricle ejection fraction at cardiac ultrasound (\%) & $44(18 \%)$ & 27 & $0.98(0.96-1.0), p=0.09$ & $\mathrm{NI}$ \\
\hline Supra-ventricular arrhythmias (yes vs. no) & 0 & 26 & $1.9(1.01-3.6), p=0.046$ & $\mathrm{NI}$ \\
\hline Septic shock (yes vs. no) & 0 & 37 & $2.1(1.2-3.7), p=0.01$ & I/NR \\
\hline Fluid balance between ICU admission and first SBT (per L) & $15(6 \%)$ & 44 & $1.07(1.03-1.12), p=0.002$ & $\mathrm{NI}$ \\
\hline Acute respiratory failure as cause of intubation (yes vs. no) & 0 & 55 & $3.0(1.7-5.2), p<0.001$ & $\mathrm{NI}$ \\
\hline $\mathrm{PaO}_{2} / \mathrm{FiO}_{2}$ ratio (per mmHg) & $3(1 \%)$ & 58 & $0.994(0.991-0.997), p<0.001$ & $0.996(0.993-1.0), p=0.03$ \\
\hline Duration of MV before the first SBT (per day) & 0 & 57 & $1.11(1.06-1.17), p<0.001$ & $1.11(1.05-1.17), p<0.001$ \\
\hline ARDS before the first SBT (yes vs. no) & 0 & 49 & $3.0(1.6-5.7), p<0.001$ & $\mathrm{NI}$ \\
\hline Neuromuscular blockade before the first SBT (yes vs. no) & 0 & 54 & $3.5(1.9-6.6), p<0.001$ & $\mathrm{NI}$ \\
\hline VAP before the first SBT (yes vs. no) & 0 & 33 & $2.6(1.2-5.4), p=0.01$ & $\mathrm{NI}$ \\
\hline Moderate-to-large pleural effusion (yes vs. no) & 0 & 58 & $3.2(1.8-5.7), p<0.001$ & $3.0(1.5-5.8), p=0.001$ \\
\hline
\end{tabular}

SAPS // simplified acute physiology score, COPD chronic obstructive pulmonary disease, ARDS acute respiratory distress syndrome, VAP ventilator-associated pneumonia, SBT spontaneous breathing trial, NI not included, I/NR included, but not retained by the final model

Among related univariate factors, only the most statistically robust (yet clinically relevant) was entered into the regression model in order to minimize the effect of colinearity. The selection process was guided by consistency (less than $5 \%$ missing values) and maximal imbalances between groups (as estimated by absolute standardized differences), as follows: cardiac disease was selected among supra-ventricular arrhythmias, left ventricle ejection fraction and cardiac disease; septic shock was selected among fluid balance between ICU admission and first SBT and septic shock; $\mathrm{PaO}_{2} / \mathrm{FiO}_{2}$ ratio was selected among acute respiratory failure as cause of intubation and $\mathrm{PaO}_{2} / \mathrm{FiO}_{2}$ ratio; duration of $\mathrm{MV}$ before the first SBT was selected among neuromuscular blockade, duration of $\mathrm{MV}$ before the first SBT, VAP, and ARDS. The multivariable model showed a good calibration as assessed by the Hosmer and Lemeshow goodness-of-fit test $\left[X^{2}(8 \mathrm{~d} f)=6.8, p=0.56\right]$ and a fair discrimination as assessed by the receiver operating characteristics curve [area under the curve of $0.76(0.69-0.82), p<0.001$ ]

usefulness and safety of reducing moderate-to-large pleural effusion at initiation of ventilator weaning, either by aggressive depletion or drainage.

\section{Additional files}

Additional file 1. Methods Supplement, Table e1, Table e2, Table e3, Table e4, Table e5.

Additional file 2. Change in interpleural distance during the 24 and $48 \mathrm{~h}$ following failure of spontaneous breathing trial according to fluid balance.

\section{Abbreviations}

SBT: spontaneous breathing trial; ARDS: acute respiratory distress syndrome; ICU: intensive care unit.

\section{Authors' contributions}

Dr Razazi had full access to all of the data in the study and takes responsibility for the integrity of the data and the accuracy of the data analysis. Dr Razazi, Dr Mekontso Dessap contributed to initial study design, analysis, interpretation of data, drafting of the submitted article, critical revisions for intellectual content. Dr Florence Boissier, Dr Mathilde Neuville, Dr Sébastien Jochmans, Dr Martial Tchir, Dr Faten May, Dr Nicolas de Prost, Dr Christian Brun-Buisson, MD, Dr Guillaume Carteaux contributed to study design and analysis, interpretation of data, drafting of the submitted article, critical revisions for intellectual content. All authors read and approved the final manuscript.

\section{Author details}

${ }^{1}$ AP-HP, DHU A-TVB, Service de Réanimation Médicale, Hôpitaux Universitaires Henri Mondor, 94010 Créteil, France. ${ }^{2}$ Faculté de Médecine de Créteil, IMRB, GRC CARMAS, Université Paris Est Créteil, 94010 Créteil, France. ${ }^{3}$ Unité U955 (Institut Mondor de Recherche Biomédicale), INSERM, Créteil, France.

${ }^{4}$ Service de Réanimation Médicale, Centre Hospitalier Universitaire de Poitiers, Poitiers 86021, France. ${ }^{5}$ AP-HP, Service de Réanimation Médicale, Hôpital Européen Georges Pompidou, 75015 Paris, France. ${ }^{6}$ AP-HP, Réanimation Médicale et des Maladies Infectieuses, Hôpital Bichat Claude Bernard, Paris, France. ${ }^{7}$ Département de Médecine Intensive, Groupe Hospitalier Sud Ile-de-France, Hôpital de Melun, 77011 Melun, France. ${ }^{8}$ Service de Réanimation, Centre Hospitalier de Villeneuve-Saint-Georges, 94190 Villeneuve-Saint-Georges, France.

\section{Acknowledgements}

This study was carried out as part of our routine clinical work.

\section{Competing interests}

The authors declare that they have no competing interests.

\section{Availability of data and materials}

The datasets generated during and/or analyzed during the current study are not publicly available as consent for publication of raw data was not obtained from study participants, but are available from the corresponding author on reasonable request.

Consent for publication

Not applicable. 


\section{Ethic approval}

This study was approved by an Institutional Review Board (CPP Ile de France $V)$, as a component of standard care, and informed consent was waived. Written and oral information about the study was given to the patients or families.

\section{Funding}

None.

\section{Publisher's Note}

Springer Nature remains neutral with regard to jurisdictional claims in published maps and institutional affiliations.

Received: 13 August 2018 Accepted: 20 October 2018

Published online: 01 November 2018

\section{References}

1. Fartoukh M, Azoulay E, Galliot R, Le Gall J-R, Baud F, Chevret S, et al. Clinically documented pleural effusions in medical ICU patients: how useful is routine thoracentesis? Chest. 2002;121:178-84.

2. Mattison LE, Coppage L, Alderman DF, Herlong JO, Sahn SA. Pleural effusions in the medical ICU: prevalence, causes, and clinical implications. Chest. 1997;111:1018-23.

3. Chiumello D, Marino A, Cressoni M, Mietto C, Berto V, Gallazzi E, et al. Pleural effusion in patients with acute lung injury: a CT scan study. Crit Care Med. 2013:41:935-44.

4. Razazi K, Thille AW, Carteaux G, Beji O, Brun-Buisson C, Brochard L, et al. Effects of pleural effusion drainage on oxygenation, respiratory mechanics, and hemodynamics in mechanically ventilated patients. Ann Am Thorac Soc. 2014;11:1018-24.

5. De Troyer A, Leduc D, Cappello M, Gevenois PA. Mechanics of the canine diaphragm in pleural effusion. J Appl Physiol Bethesda Md. 1985;2012(113):785-90.

6. Estenne M, Yernault JC, De Troyer A. Mechanism of relief of dyspnea after thoracocentesis in patients with large pleural effusions. Am J Med. 1983:74:813-9.

7. Goligher EC, Leis JA, Fowler RA, Pinto R, Adhikari NKJ, Ferguson ND. Utility and safety of draining pleural effusions in mechanically ventilated patients: a systematic review and meta-analysis. Crit Care Lond Engl. 2011;15:R46.

8. Esteban A, Alía I, Ibañez J, Benito S, Tobin MJ. Modes of mechanical ventilation and weaning. A national survey of Spanish hospitals. The Spanish lung failure collaborative group. Chest. 1994;106:1188-93.

9. Dres M, Roux D, Pham T, Beurton A, Ricard J-D, Fartoukh M, et al. Prevalence and impact on weaning of pleural effusion at the time of liberation from mechanical ventilation: a multicenter prospective observational study. Anesthesiology. 2017;126:1107-15.

10. Boles J-M, Bion J, Connors A, Herridge M, Marsh B, Melot C, et al. Weaning from mechanical ventilation. Eur Respir J. 2007;29:1033-56.

11. Girault C, Bubenheim M, Abroug F, Diehl JL, Elatrous S, Beuret P, et al. Noninvasive ventilation and weaning in patients with chronic hypercapnic respiratory failure: a randomized multicenter trial. Am J Respir Crit Care Med. 2011;184:672-9.
12. Thille AW Boissier F, Ben-Ghezala H, Razazi K, Mekontso-Dessap A, BrunBuisson C, et al. Easily identified at-risk patients for extubation failure may benefit from noninvasive ventilation: a prospective before-after study. Crit Care Lond Engl. 2016;20:48.

13. Béduneau G, Pham T, Schortgen F, Piquilloud L, Zogheib $E_{\text {t }}$ Jonas M, et al. Epidemiology of Weaning Outcome according to a New Definition. The WIND Study. Am J Respir Crit Care Med. 2017;195:772-83.

14. Mekontso Dessap A, Roche-Campo F, Kouatchet A, Tomicic V, Beduneau $G$, Sonneville $R$, et al. Natriuretic peptide-driven fluid management during ventilator weaning: a randomized controlled trial. Am J Respir Crit Care Med. 2012;186:1256-63.

15. Yang PC, Luh KT, Chang DB, Wu HD, Yu CJ, Kuo SH. Value of sonography in determining the nature of pleural effusion: analysis of 320 cases. AJR Am J Roentgenol. 1992;159:29-33.

16. Volpicelli G, Elbarbary M, Blaivas M, Lichtenstein DA, Mathis G, Kirkpatrick AW, et al. International evidence-based recommendations for point-ofcare lung ultrasound. Intensive Care Med. 2012;38:577-91.

17. Balik M, Plasil P, Waldauf P, Pazout J, Fric M, Otahal M, et al. Ultrasound estimation of volume of pleural fluid in mechanically ventilated patients. Intensive Care Med. 2006:32:318-21.

18. Lichtenstein D, Hulot JS, Rabiller A, Tostivint I, Mezière G. Feasibility and safety of ultrasound-aided thoracentesis in mechanically ventilated patients. Intensive Care Med. 1999;25:955-8.

19. Austin PC. Balance diagnostics for comparing the distribution of baseline covariates between treatment groups in propensity-score matched samples. Stat Med. 2009;28:3083-107

20. Havelock T, Teoh R, Laws D, Gleeson F, BTS Pleural Disease Guideline Group. Pleural procedures and thoracic ultrasound: British Thoracic Society Pleural Disease Guideline 2010. Thorax. 2010;65(Suppl 2):ii61-76.

21. Walker SP, Morley AJ, Stadon L, De Fonseka D, Arnold DA, Medford AR, et al. Non-malignant pleural effusions (NMPE): a prospective study of 356 consecutive unselected patients. Chest. 2017;151(5):1099-105.

22. Mccabe WR, Jackson G. Gram-negative bacteremia: I. etiology and ecology. Arch Intern Med. 1962;110:847-55.

23. Light RW. Clinical practice. Pleural effusion. N Engl J Med. 2002:346:1971-7.

24. Umbrello M, Mistraletti G, Galimberti A, Piva IR, Cozzi O, Formenti P. Drainage of pleural effusion improves diaphragmatic function in mechanically ventilated patients. Crit Care Resusc J Australas Acad Crit Care Med. 2017;19:64-70.

25. Mayo P, Volpicelli G, Lerolle N, Schreiber A, Doelken P, Vieillard-Baron A. Ultrasonography evaluation during the weaning process: the heart, the diaphragm, the pleura and the lung. Intensive Care Med. 2016:42:1107-17.

26. Begot E, Grumann A, Duvoid T, Dalmay F, Pichon N, François B, et al. Ultrasonographic identification and semiquantitative assessment of unloculated pleural effusions in critically ill patients by residents after a focused training. Intensive Care Med. 2014;40:1475-80

27. Giglioli C, Spini V, Landi D, Chiostri M, Romano SM, Calabretta R, et al. Congestive heart failure and decongestion ability of two different treatments: continuous renal replacement and diuretic therapy: experience of a cardiac step down unit. Acta Cardiol. 2013;68:355-64. 